\title{
The Indian Institute of Science, Bangalore
}

$\mathrm{I}^{\mathrm{N}}$ 1926 the Government of India appointed a committee with Sir William Pope as chairman to report upon the Indian Institute of Science, and one of its recommendations was that the activities of the Institute should be subject to review by a committee every five years. Early this year, Sir James Irvine was appointed chairman of the statutory quinquennial committee, and the issue of the report of this committee is awaited with interest. It is untimely, therefore, that the March issue of our Calcutta contemporary, Science and Culture, should publish a severely critical article on the present administration of the Institute. Anyone cognisant of the large volume of original work which has issued from the Institute since its foundation cannot doubt that it has more than justified the hopes of its munificent founder, the late Mr. J. N. Tata. The two main heads of the recent criticisms would appear to be $(a)$ that the work of the Institute is too academic and $(b)$ that, since the students are drawn very largely from South India, it is no longer an all-India research institute.

Whilst it may be true that much of the work carried out in the laboratories of the Institute is concerned mainly with problems of purely scientifie interest, it is difficult to see how this could be otherwise. The research work in the Institute is done by young students who go there for a training in the methods of research. If they are not to be dis. couraged in their early years, it is essential that they should be assigned problems likely to yield results within a reasonable time. Furthermore, we very much doubt if it is possible to undertake ad hoc research on industrial problems. The industrial research institutions in Great Britain work in close contact with established industries, and they study fundamental problems related to these. It is very rare indeed that a new industry results directly from an isolated piece of research. We are of opinion that the Indian Institute of Science can best assist industrial development by working in collaboration with industry, as do the universities in Great Britain with Imperial Chemical Industries, Ltd.

The contributions of the Institute to industrial de. velopment have not been negligible; we need only instance the valuable researches made in collaboration with the Forest Research Institute at Dehra Dun on the causation of sandal wood disease, the investigations on wireless transmission in the tropics, and the work leading to the establishment of the sandalwood oil and white lead industries.

All industrial advancement is dependent upon pure scientific research, and it is a matter for congratulation that Sir C. V. Raman, the present director, has already built up a school of research in physics and that he was able to attract as a visitor to Bangalore so eminent a theoretical physicist as Dr. Max Born. Whilst the rapid development of research in Indian universities, not foreseen by its founders, has doubtless made necessary a new orientation of the activities of the Institute, we cannot doubt that it will continue to play a great part in the scientific advancement of India.

\section{Progress in Atomic Physics}

\section{Conference at COPEnhagen}

$\mathrm{P}$ ROF. NIELS BOHR held a conference on atomic physics on June $17-20$ in the Institute for Theoretical Physics at Copenhagen. About eighty physicists attended the discussions, including many from foreign countries.

The outstanding communication to the conference was a paper by $W$. Heisenberg on the quantum. mechanical theory of cosmic ray showers. Up to the present, no explanation of the fact that particles of very high energy can produce simultaneously, or in a single process, a large number of secondary particles, has been given in terms of the quantum theory of electrodynamics. Such showers of secondary particles have been observed by Hoffmann, Blackett and other experimenters.

According to quantum electrodynamics, the effect. ive cross-sections for the simultaneous formation of $n$ pairs of electrons lead to an extremely small prob. ability for the occurrence of large showers. This calculation was made on the assumption that only the ordinary known electrical forces are of importance in the interaction between charged particles.
The phenomenon of $\beta$-disintegration has, however, suggested the idea, as in Fermi's theory, that there exist interaction forces of a new type between electrons and heavy elementary particles such as protons and neutrons. In this new type of interaction, a fourth elementary particle, the neutrino, probably takes part, as has been suggested by Pauli. Without the neutrino, it is impossible to preserve the law of the conservation of energy in the description of $\beta$-dis. integration. It follows from the existence of these new interaction forces that an electron and neutrino can be created in an atomic nucleus, the electron being emitted as a $\beta$-ray, as Fermi pointed out.

Heisenberg has given a general proof that all inter. action forces of the type that produce $\beta$-disintegration lead to the production of multiple processes and particles, when initiated by partieles of high energy. The process may be of the following sort. A high. speed proton strikes a nucleus and produces in the field of the nucleus a great number of $\beta$-rays in one elementary action. Heisenberg suggests that the mechanism of the cosmic ray showers is of this type. 